Polymer Journal, Vol. 39, No. 4, pp. 379-388 (2007)

(C) 2007 The Society of Polymer Science, Japan

\title{
Precise Synthesis of Poly(silphenylenesiloxane)s with Epoxy Side Functional Groups by Tris(pentafluorophenyl)borane as a Catalyst
}

\author{
Lei XUE and Yusuke KAWAKAMI ${ }^{\dagger}$ \\ Graduate School of Materials Science, Japan Advanced Institute of Science and Technology, \\ Asahidai 1-1, Nomi 923-1292, Japan
}

(Received November 24, 2006; Accepted January 24, 2007; Published March 5, 2007)

\begin{abstract}
Dimethylhydroxysilyl, -dimethylhydrosilyl, -dimethylmethoxysilyl -terminated tetramethylsilphenylenesiloxane prepolymers were prepared by sequential palladium-catalyzed dehydrocoupling polymerization of 1,4-bis(dimethylsilyl)benzene with water, reduction with lithium aluminium hydride, and palladium-catalyzed methanolysis. 1,4-Bis[(glycidyl- or cyclohexene oxide-functionalized alkyl)methylhydrosilyl]benzene and methyldimethoxysilanes with the same epoxy groups were synthesized by hydrosilylation of 1,4-bis(methyldihydrosilyl)benzene and methyldimethoxysilane with (terminal olefin-functionalized alkyl) substituted epoxides in the presence of platinum catalyst. 1,4-Bis[(glycidyl- or cyclohexene oxide-functionalized alkyl)methyl(hydroxy or methoxy)silyl]benzenes were obtained by hydrolysis and methanolysis of the hydrosilyl derivatives. Poly(silphenylenesiloxane)s with epoxy side groups were synthesized by hetero-polycondensation of the hydrosilane, hydroxysilane and methoxysilane functional groups in the prepolymer and 1,4-bis[(epoxy-functionalized alkyl)methylsilyl]benzene, or 1,1-dimethoxy(epoxy-functionalized alkyl)methylsilane in the presence of a catalyst. Tris(pentafluorophenyl)borane was found to be an excellent catalyst for the polymerization. Cross-linking a polymer with 1,4-diaminobenzene gave stretchable polymer film by solution cast method. [doi:10.1295/polymj.PJ2006171]

KEY WORDS Palladium Catalyst / Tris(pentafuluorophenyl)borane / Hydrosilylation / Dehydrocoupling Polymerization / Poly(silphenylenesiloxane) / Epoxy Side Groups / Cross-Linked Polymer Film /
\end{abstract}

Modern technologies have continuous demands on development of high-temperature elastomeric materials. Polysiloxanes are a kind of good candidates as high-temperature elastomer because of the property originated from flexible and heat-resistant $\mathrm{Si}-\mathrm{O}-\mathrm{Si}$ backbone, which is also the origin of good dielectric and surface properties, and so on. ${ }^{1-3}$

However, some thermal degradation reaction of polysiloxanes was noticed at high temperature. Cor$\mathrm{riu}^{4}$ elucidated that the main course of degradation of polysiloxane is the intermolecular exchanges of $\mathrm{Si}-\mathrm{O}$ bonds, followed by intramolecular exchanges of $\mathrm{Si}-\mathrm{O}$ bonds to depolymerize. Incorporation of rigid aromatic units into the siloxane backbone generally interrupts the siloxane redistribution reaction at high temperature, and improves both mechanical and thermal properties without simultaneous loss of other good features of polysiloxanes. Thus, polysilarylenesiloxanes, derivatives of polysiloxanes by partially replacing -O- by arylene groups, show better thermal and mechanical property than polysiloxanes. ${ }^{2,3,5,6}$

Polysilphenylenesiloxanes, a class of typical polysilarylenesiloxane, were intensively reported as materials possessing thermal stability and satisfying low temperature elasticity, although their crystallinity is usually higher than polysiloxanes, which is a possible obstacle in various applications, for instance, application in electronic device materials as a thin film. One of the attractive aspects of these polymers is the easiness of introducing variety of functional substituents, such as vinyl, phenyl, hydrido, flouroalkyl, etc. ${ }^{7-11}$ These functional groups were found to significantly affect the thermal property and crystallinity of polymers.

Meanwhile, cross-linked materials have many superior properties as elastomers. ${ }^{12}$ Epoxy resins have found use in a wide variety of applications due to their desirable properties, such as high strength and modulus properties, excellent adhesive property, good thermal-mechanical and electrical properties and adjustment of crystallinity. Although generally hard and relatively brittle nature of the epoxy resins has limited the application of the materials in many fields, they have been utilized as insulation materials. ${ }^{13}$

The properties of elastomers, such as silicone rubber and poly(tetramethylsilphenylenesiloxane) (PTMPS), thus precisely modified with epoxy group will find a good chance to be used as insulating materials because of the improved properties. ${ }^{14-16}$

However, efficient synthetic methods of PTMPS's

${ }^{\dagger}$ To whom correspondence should be addressed (Tel: +81-761-51-1630, Fax: +81-761-51-1635, E-mail: kawakami@jaist.ac.jp). 
having epoxy side groups with precisely regulated sequence have not been established. In this research, we were dedicated to the development of new synthetic method to obtain PTMPS having epoxy side groups with regulated interval along the main chain. Special stress was given to the use of tris(pentafluorophenyl)borane as excellent catalyst to form siloxane bond from silane and methoxysilane or hydroxysilane, by suppressing homo-condensation of hydroxysilane.

Cross-linking of the epoxy groups by 1,4-diaminobenzene was also carried out, and change in thermal degradation behavior was briefly discussed.

\section{EXPERIMENTAL}

\section{Measurements}

${ }^{1} \mathrm{H}$ NMR spectra were obtained in $\mathrm{CDCl}_{3}$ on a Varian $500 \mathrm{MHz}$ spectrometer model Unity INOVA. Chemical shifts are reported in ppm relative to $\mathrm{CHCl}_{3}$ ( $\delta$ 7.26). Gel permeation chromatography (GPC) analysis was performed on a JASCO HPLC Gulliver 900 equipped with a UV detector on the combination of Shodex KF-801 (exclusion limit: polystyrene, $1.5 \times$ $10^{3}$ dalton) and KF-802 (exclusion limit: polystyrene, $5.0 \times 10^{3}$ dalton), or KF-803L (exclusion limit: polystyrene, $7.0 \times 10^{4}$ dalton) and KF-804 (exclusion limit: polystyrene, $4.0 \times 10^{5}$ dalton) columns using tetrahydrofuran (THF) as an eluent (flow rate, $1 \mathrm{~mL} / \mathrm{min}$ ). The differential scanning calorimetric (DSC) analysis was performed on a Seiko DSC6200 instrument at a heating rate of $10.0^{\circ} \mathrm{C} / \mathrm{min}$ in air. The thermogravimetric analysis (TGA) was performed on a Seiko Instruments TGA/DTA 220 with a heating rate of $10.0^{\circ} \mathrm{C} / \mathrm{min}$ under nitrogen flow or in air. IR spectra were obtained on a JASCO VALOR-III spectrophotometer.

\section{Materials}

Methyldichlorosilane and dimethoxymethylsilane were obtained from Shin-Etsu Chemical. 1,4-Dibromobenzene, allyl glycidyl ether (AGE), 1,2-epoxy5-hexene (EH), 4-vinyl-1-cyclohexene-1,2-epoxide (VCHE), platinum(0)-1,3-divinyl-1,1,3,3-tetramethyldisiloxane complex in xylene $\left(\operatorname{Pt}(0)(\mathrm{DVTMDS})_{2}\right)$, di- $n$-butyltin dilaurate, tris(dibenzylideneacetone)dipalladium(0)-chloroform adduct $\left(\mathrm{Pd}_{2}(\mathrm{dba})_{3} \cdot \mathrm{CHCl}_{3}\right)$, and tris(pentafluorophenyl)borane were purchased from Sigma-Aldrich. Lithium aluminum hydride (LAH) was purchased from Kanto Chemical Co. Inc. 1,4-diaminobenzene was obtained from Nacalai Co. Inc. 1,4-Bis(dimethylsilyl)benzene (BSB) and PTMPS were prepared according to the literatures. ${ }^{17-19}$ Hexane, tetrahydrofuran (THF), methanol and diethyl ether were dried before use.
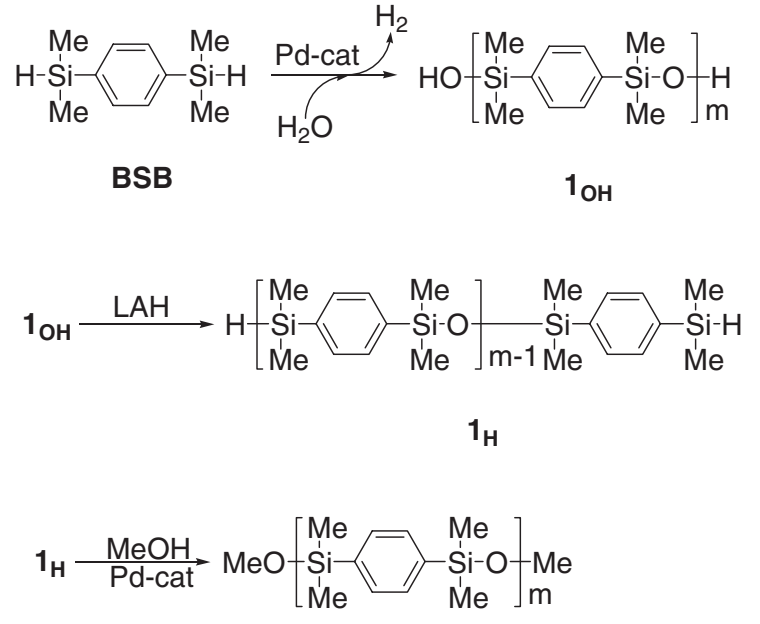

$$
1 \text { OMe }
$$

Scheme 1.

\section{Synthesis of Prepolymer and Monomers}

The synthetic pathways of prepolymers with bis(hydroxysilyl, hydrosilyl and methoxylsilyl) terminal groups, needed for the synthesis of polymers in Scheme 3, are shown in Scheme 1.

\section{Poly(silphenylenedisiloxane) Prepolymer (10H)}

To a $200 \mathrm{~mL}$ Schlenk flask were added $\mathrm{Pd}_{2}(\mathrm{dba})_{3}$. $\mathrm{CHCl}_{3}(0.15 \mathrm{~g}, 0.15 \mathrm{mmol}), \mathrm{BSB}(26.8 \mathrm{~g}, 0.15 \mathrm{~mol})$ and THF $(60 \mathrm{~mL})$ in argon atmosphere. Distilled water $(2.97 \mathrm{~g}, 0.17 \mathrm{~mol})$ was added slowly with a syringe at room temperature. Hydrogen gas evolution occurred and ceased. After stirring for $5 \mathrm{~h}$, the reaction system was exposed to air and stirred for $3 \mathrm{~h}$ to destroy the catalyst. The decomposed catalyst was removed by filtration through a short Florisil column using chloroform as an eluent. Fractional precipitation was carried out by adding saturated toluene solution of the polymeric products into methanol as a poor solvent to obtain the prepolymer $\mathbf{1 0 H}$.

Yield: 28.5 g. $M_{\mathrm{n}}=2,800, M_{\mathrm{w}} / M_{\mathrm{n}}=1.04 . \delta: 0.32(\mathrm{~s}$, $\left.\mathrm{SiCH}_{3}\right), 7.53\left(\mathrm{~s}, \mathrm{C}_{6} \mathrm{H}_{4}\right) . \mathrm{E}_{\mathrm{LEM}} \cdot \mathrm{A}_{\mathrm{NAL}} \cdot$ Calcd for $\left(\mathrm{C}_{10^{-}}\right.$ $\left.\mathrm{H}_{16} \mathrm{Si}_{2} \mathrm{O}\right): \mathrm{C}, 57.63 \%, \mathrm{H}, 7.74 \%$. Found: C, $57.51 \%$, $\mathrm{H}, 7.70 \%$.

\section{Poly(silphenylenedisiloxane) Prepolymer (1H)}

A solution of prepolymer $10 \mathrm{H}(22.0 \mathrm{~g}, 15.7 \mathrm{mmol}$ $-\mathrm{OH})$ in THF $(50 \mathrm{~mL})$ was slowly added to LAH $(0.60 \mathrm{~g}, 15.7 \mathrm{mmol})$ in THF $(10 \mathrm{~mL})$ solution at an ice-water bath temperature. After the addition, the reaction mixture was warmed to room temperature, and stirred for $18 \mathrm{~h}$. The product mixture was diluted with ether $(50 \mathrm{~mL})$, and $1 \mathrm{~N} \mathrm{HCl}$ was slowly added at icewater bath temperature. The organic layer was washed with distilled water $(30 \mathrm{~mL}$ each) for three times in a separatory funnel, and dried over anhydrous sodium 
sulfate. Evaporation of the solvent and precipitation in methanol gave the product.

Yield: $68 \% . M_{\mathrm{n}}=2,700\left(2,720\right.$ by ${ }^{1} \mathrm{H}$ NMR $), M_{\mathrm{w}} /$ $M_{\mathrm{n}}=1.04 . \quad \delta: 0.33\left(\mathrm{~s}, \mathrm{Si}\left(\mathrm{CH}_{3}\right)_{2}\right), 0.40(\mathrm{~d}, J=3.7$ $\left.\mathrm{Hz}, 12 \mathrm{H}, \mathrm{HSi}\left(\mathrm{CH}_{3}\right)_{2}\right), 4.42(\mathrm{~h}, J=3.7 \mathrm{~Hz}, 2 \mathrm{H}, \mathrm{Si} H)$, $7.54\left(\mathrm{~s}, \mathrm{C}_{6} \mathrm{H}_{4}\right)$. $\mathrm{E}_{\mathrm{LEM}}$. $\mathrm{A}_{\mathrm{NAL}}$. Calcd for $\left(\mathrm{C}_{10} \mathrm{H}_{16} \mathrm{Si}_{2} \mathrm{O}\right)$ : C, $57.63 \%$, H, 7.74\%. Found: C, 57.40\%, H, 7.80\%.

\section{Poly(silphenylenedisiloxane) Prepolymer (1OMe)}

Methanol $(0.40 \mathrm{~g}, 12.5 \mathrm{mmol})$ was added to the solution of prepolymer $1 \mathrm{H}(6.75 \mathrm{~g}, 5.00 \mathrm{mmol}-\mathrm{H})$ in THF $(30 \mathrm{~mL})$ and $\mathrm{Pd}_{2}(\mathrm{dba})_{3} \cdot \mathrm{CHCl}_{3}(0.005 \mathrm{~g}, 5.00$ $\mu \mathrm{mol})$. After stirring for $10 \mathrm{~h}$, the catalyst was separated by a short Florisil column using chloroform as an eluent. After evaporation of the solvent, the product was re-dissolved in a small amount of toluene, and precipitated into methanol.

Yield: $56 \% . M_{\mathrm{n}}=2,900, M_{\mathrm{w}} / M_{\mathrm{n}}=1.03 . \delta: 0.33(\mathrm{~s}$, $\left.\mathrm{Si}\left(\mathrm{CH}_{3}\right)_{2}\right), 3.44$ (s, 6H, $\left.\mathrm{SiOCH}_{3}\right), 7.54\left(\mathrm{~s}, \mathrm{C}_{6} \mathrm{H}_{4}\right)$.

Synthesis of comonomers for polycondensation is shown in Scheme 2.

\section{1,4-Bis(methylchlorosilyl)benzene (2)}

To the mixture of methyldichlorosilane $(103.7 \mathrm{~g}$, $0.90 \mathrm{~mol})$ and magnesium turning $(10.9 \mathrm{~g}, 0.45 \mathrm{~mol})$ in THF $(60 \mathrm{~mL}), 1,4$-dibromobenzene $(35.4 \mathrm{~g}, 0.15$ mol) in THF $(50 \mathrm{~mL})$ was slowly added, and stirred for $8 \mathrm{~h}$ at room temperature. The yellowish crude product, obtained as the hexane extract after evaporation of THF, was purified by fractional distillation to obtain the pure product as colorless liquid.

Yield: $42 \%\left(63^{\circ} \mathrm{C} / 65 \mathrm{~Pa}\right) . \delta: 0.78(\mathrm{~d}, J=3.1 \mathrm{~Hz}, 6 \mathrm{H}$, $\left.\mathrm{SiCH}_{3}\right), 5.32(\mathrm{q}, J=3.1 \mathrm{~Hz}, 2 \mathrm{H}, \mathrm{SiH}), 7.71(\mathrm{~s}, 4 \mathrm{H}$, $\mathrm{C}_{6} \mathrm{H}_{4}$ ).

\section{1,4-Bis(methylsilyl)benzene (3)}

Compound $2(13.5 \mathrm{~g}, 57.4 \mathrm{mmol})$ was diluted with diethyl ether $(30 \mathrm{~mL})$, and added slowly to a flask containing LAH $(2.18 \mathrm{~g}, 57.4 \mathrm{mmol})$ in diethyl ether $(20 \mathrm{~mL})$ solution. After stirring for $20 \mathrm{~h}$ at room temperature, a small amount of concentrated $\mathrm{HCl}$ was slowly added to the mixture that was diluted with ether $(30 \mathrm{~mL})$ at an ice-bath temperature. The organic layer was washed three times with distilled water $(20 \mathrm{~mL}$ each) in a separatory funnel, dried over sodium sulfate, and evaporated to remove the solvents. Distillation under reduced pressure gave a colorless liquid.

Yield: $52 \%\left(26^{\circ} \mathrm{C} / 66 \mathrm{~Pa}\right) . \delta: 0.45(\mathrm{t}, J=4.2 \mathrm{~Hz}, 6 \mathrm{H}$, $\mathrm{SiCH}_{3}$ ), 4.36 (q, $\left.J=4.2 \mathrm{~Hz}, 4 \mathrm{H}, \mathrm{SiH}\right), 7.60$ (s, $4 \mathrm{H}$, $\mathrm{C}_{6} \mathrm{H}_{4}$ ).

\section{1,4-Bis[(epoxy-functionalizedalkyl)methylsilyl]benzenes $(4 \mathrm{H}, \mathrm{a}-\mathrm{c})$}

A solution of $\operatorname{Pt}(0)(\mathrm{DVTMDS})_{2}$ in xylene $(0.01 \mathrm{~g}$,

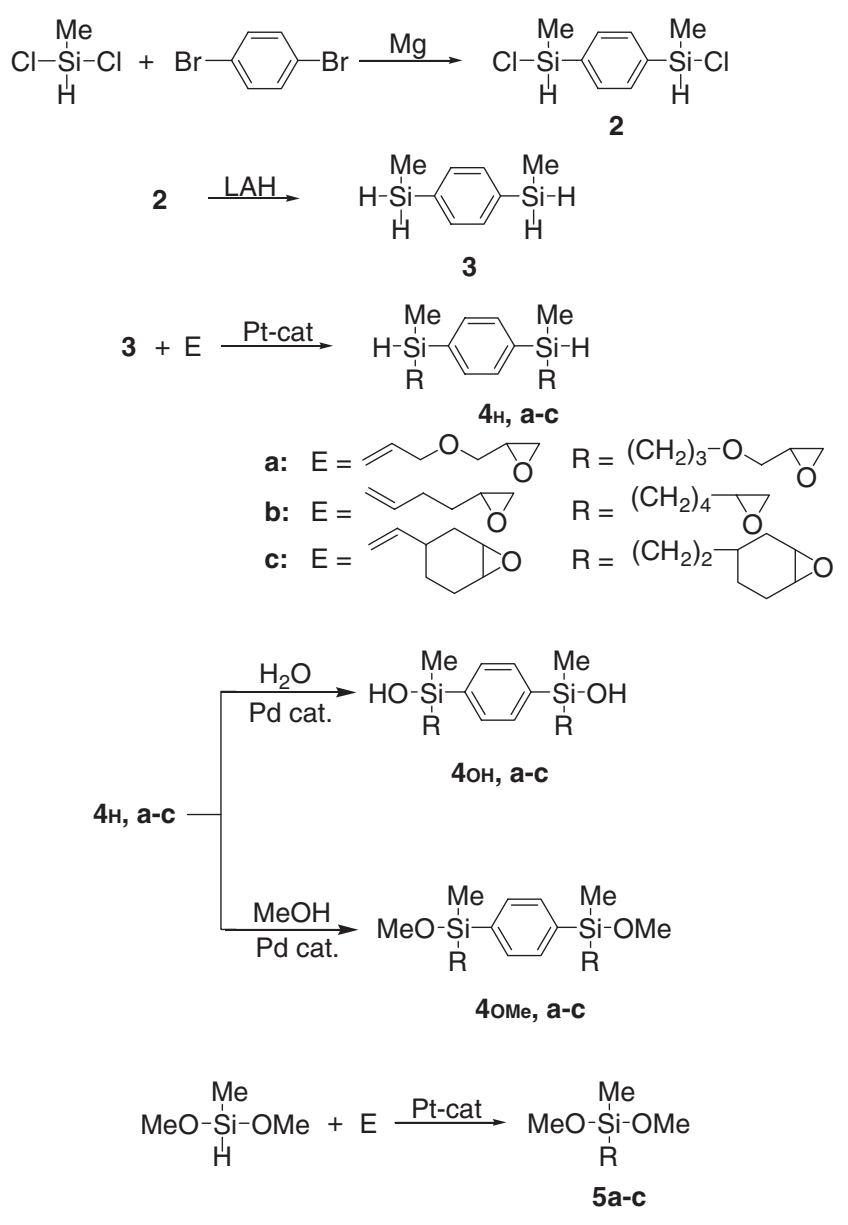

Scheme 2.

$27.0 \mu \mathrm{mol})$ was added to a mixture of $3(4.50 \mathrm{~g}$, $27.0 \mathrm{mmol})$ and AGE or EH or VCHE $(27.0 \mathrm{mmol})$ in THF. After stirring at $60^{\circ} \mathrm{C}$, the catalyst was separated by a short Florisil column. The products were isolated by silica gel column chromatography using toluene/hexane $(2 / 1, \mathrm{v} / \mathrm{v})$ as the eluent.

4H,a: Yield: $46 \%(\mathrm{Rf}=0.2) . \delta: 0.36(\mathrm{~d}, J=6.7 \mathrm{~Hz}$, $\left.6 \mathrm{H}, \mathrm{SiCH}_{3}\right), 0.77\left(\mathrm{~m}, 4 \mathrm{H}, \mathrm{SiCH}_{2}\right), 1.59(\mathrm{~m}, 4 \mathrm{H}, \mathrm{Si}-$ $\left.\mathrm{CH}_{2} \mathrm{CH}_{2}\right),[2.58(\mathrm{q}, J=2.7 \mathrm{~Hz}), 2.77(\mathrm{q}, J=4.6$ $\mathrm{Hz}), 4 \mathrm{H}, \mathrm{CH}_{2}$ in epoxy ring], $3.17(\mathrm{~m}, 2 \mathrm{H}, \mathrm{CH}$ in epoxy ring), [3.33 (q, $J=5.7 \mathrm{~Hz}), 3.66(\mathrm{q}, J=3.0 \mathrm{~Hz})$, $\left.4 \mathrm{H}, \mathrm{Si}\left(\mathrm{CH}_{2}\right)_{3} \mathrm{OCH}_{2}\right], 3.41\left(\mathrm{~m}, 4 \mathrm{H}, \mathrm{Si}\left(\mathrm{CH}_{2}\right)_{2} \mathrm{CH}_{2} \mathrm{O}\right)$, $4.82(\mathrm{q}, J=6.7 \mathrm{~Hz}, 2 \mathrm{H}, \mathrm{Si} H), 7.47\left(\mathrm{~s}, 4 \mathrm{H}, \mathrm{C}_{6} H_{4}\right)$. Calculated molecular weight: 394.6572 . EI-MS $(\mathrm{m} / z)$ : $394.6571\left([\mathrm{M}]^{+}\right), 393.6493\left([\mathrm{M}-\mathrm{H}]^{+}\right]$.

4H,b: Yield: $61 \%(\mathrm{Rf}=0.4) . \delta: 0.35(\mathrm{~d}, J=6.1 \mathrm{~Hz}$, $\left.6 \mathrm{H}, \mathrm{SiCH}_{3}\right), 0.72\left(\mathrm{~m}, 4 \mathrm{H}, \mathrm{SiCH}_{2}\right), 1.49-1.70(\mathrm{~m}, 12 \mathrm{H}$, $\left.\mathrm{SiCH}_{2}\left(\mathrm{CH}_{2}\right)_{3}\right),\left[2.43(\mathrm{q}, J=2.7 \mathrm{~Hz}), 2.72\left(\mathrm{q}, J_{2}=\right.\right.$ $3.9 \mathrm{~Hz}), 4 \mathrm{H}, \mathrm{CH}_{2}$ in epoxy ring], $2.89(\mathrm{~m}, 2 \mathrm{H}, \mathrm{CH}$ in epoxy ring), 4.35 (q, $J=6.1 \mathrm{~Hz}, 2 \mathrm{H}, \mathrm{Si} H), 7.53$ (s, $\left.4 \mathrm{H}, \mathrm{C}_{6} H_{4}\right)$.

4H,c: Yield: $33 \%(\mathrm{Rf}=0.3) . \delta: 0.37(\mathrm{~d}, J=6.2 \mathrm{~Hz}$, $\left.6 \mathrm{H}, \mathrm{SiCH}_{3}\right), 0.73,\left(\mathrm{~m}, 4 \mathrm{H}, \mathrm{SiCH}_{2}\right), 1.10-2.20$ (m, $18 \mathrm{H}, \mathrm{SiCH}_{2} \mathrm{CH}_{2}$ and $\mathrm{CH}_{2} \mathrm{CH}$ in cyclohexane ring), 
$3.12(\mathrm{~m}, 4 \mathrm{H}, \mathrm{CH}$ in epoxy ring), $4.53(\mathrm{q}, J=6.2 \mathrm{~Hz}$, $2 \mathrm{H}, \mathrm{Si} H), 7.49$ (s, 4H, $\left.\mathrm{C}_{6} H_{4}\right)$.

1,4-Bis[(epoxy-functionalized alkyl)methylsilyl]benzenes with Hydroxyl and Methoxyl Terminal Groups $(4 \mathrm{H}, \mathrm{a}-\mathrm{c}$ and $4 \mathrm{OMe}, \mathrm{a}-\mathrm{c})$

Hydrolysis or methanolysis, similarly to the case of prepolymer, changed the terminal groups of the compounds $4 H, a-c$ to $\mathrm{OH}(4 O H, a-c)$ or OMe $(4 O M e, a-c)$ groups. The products were also purified by column chromatography.

4OH,a: Yield: $51 \%(\mathrm{Rf}=0.4) . \delta: 0.42\left(\mathrm{~s}, 6 \mathrm{H}, \mathrm{SiCH}_{3}\right)$, $0.73\left(\mathrm{t}, J=8.6 \mathrm{~Hz}, 4 \mathrm{H}, \mathrm{SiCH}_{2}\right), 1.56\left(\mathrm{~m}, 4 \mathrm{H}, \mathrm{SiCH}_{2}-\right.$ $\left.\mathrm{CH}_{2}\right), 2.11(\mathrm{~s}, 2 \mathrm{H}, \mathrm{SiOH}),[2.60(\mathrm{q}, J=2.6 \mathrm{~Hz}), 2.72$ $(\mathrm{q}, J=4.1 \mathrm{~Hz}), 4 \mathrm{H}, \mathrm{CH}_{2}$ in epoxy ring], $3.18(\mathrm{~m}, 2 \mathrm{H}$, $\mathrm{C} H$ in epoxy ring), $[3.33(\mathrm{q}, J=5.9 \mathrm{~Hz}), 3.67(\mathrm{q}, J=$ $\left.3.0 \mathrm{~Hz}), 4 \mathrm{H}, \mathrm{Si}\left(\mathrm{CH}_{2}\right)_{3} \mathrm{OCH}_{2}\right], 3.39\left(\mathrm{~m}, 4 \mathrm{H}, \mathrm{Si}\left(\mathrm{CH}_{2}\right)_{2}-\right.$ $\left.\mathrm{CH}_{2} \mathrm{O}\right), 7.51$ (s, $4 \mathrm{H}, \mathrm{C}_{6} \mathrm{H}_{4}$ ). Calculated molecular weight: 426.6560. EI-MS $(\mathrm{m} / \mathrm{z})$ : $426.6465\left([\mathrm{M}]^{+}\right)$, $409.6389\left([\mathrm{M}-\mathrm{OH}]^{+}\right)$.

4oH,b: Yield: $71 \%(\mathrm{Rf}=0.6) . \delta: 0.34\left(\mathrm{~s}, 6 \mathrm{H}, \mathrm{SiCH}_{3}\right)$, $0.75\left(\mathrm{t}, J=8.1 \mathrm{~Hz}, 4 \mathrm{H}, \mathrm{SiCH}_{2}\right), 1.47-1.70(\mathrm{~m}, 12 \mathrm{H}$, $\left.\mathrm{SiCH}_{2}\left(\mathrm{CH}_{2}\right)_{3}\right), 2.09$ (s, 2H, SiOH), [2.44 (q, $J=2.7$ $\mathrm{Hz}), 2.76\left(\mathrm{q}, J=3.7 \mathrm{~Hz}, 4 \mathrm{H}, \mathrm{CH}_{2}\right.$ in epoxy ring], $2.88\left(\mathrm{~m}, 2 \mathrm{H}, \mathrm{CH}\right.$ in epoxy ring), $7.53\left(\mathrm{~s}, 4 \mathrm{H}, \mathrm{C}_{6} \mathrm{H}_{4}\right)$. 4oH,c: Yield: $47 \%(\mathrm{Rf}=0.5) . \delta: 0.35\left(\mathrm{~s}, 6 \mathrm{H}, \mathrm{SiCH}_{3}\right)$, $0.77,\left(\mathrm{t}, J=8.3 \mathrm{~Hz}, 4 \mathrm{H}, \mathrm{SiCH}_{2}\right), 1.09-2.16(\mathrm{~m}, 18 \mathrm{H}$, $\mathrm{SiCH}_{2} \mathrm{CH}_{2}$ and $\mathrm{CH}_{2} \mathrm{CH}$ in cyclohexane ring), 2.18 (s, 2H, $\mathrm{SiOH}$ ), 3.09 (m, 4H, $\mathrm{CH}$ in epoxy ring), 7.49 (s, $\left.4 \mathrm{H}, \mathrm{C}_{6} \mathrm{H}_{4}\right)$.

4OMe, a: Yield: $44 \%(\mathrm{Rf}=0.2) . \delta: 0.39(\mathrm{~s}, 6 \mathrm{H}, \mathrm{Si}-$ $\left.\mathrm{CH}_{3}\right), 0.83$ (t, $\left.J=8.3 \mathrm{~Hz}, 4 \mathrm{H}, \mathrm{SiCH}_{2}\right), 1.59(\mathrm{~m}, 4 \mathrm{H}$, $\left.\mathrm{SiCH}_{2} \mathrm{CH}_{2}\right),[2.55(\mathrm{q}, J=2.7 \mathrm{~Hz}), 2.71(\mathrm{q}, J=4.5$ $\mathrm{Hz}), 4 \mathrm{H}, \mathrm{CH}_{2}$ in epoxy ring], $3.17(\mathrm{~m}, 2 \mathrm{H}, \mathrm{CH}$ in epoxy ring), [3.34 (q, $J=6.1 \mathrm{~Hz}), 3.66(\mathrm{q}, J=3.2 \mathrm{~Hz})$, $\left.4 \mathrm{H}, \mathrm{Si}\left(\mathrm{CH}_{2}\right)_{3} \mathrm{OCH}_{2}\right], 3.42\left(\mathrm{~m}, 4 \mathrm{H}, \mathrm{Si}\left(\mathrm{CH}_{2}\right)_{2} \mathrm{CH}_{2} \mathrm{O}\right)$, $3.55\left(\mathrm{~s}, 6 \mathrm{H}, \mathrm{SiOCH}_{3}\right), 7.43$ (s, $\left.4 \mathrm{H}, \mathrm{C}_{6} \mathrm{H}_{4}\right)$. Calculated molecular weight: 454.7096 . EI-MS $(\mathrm{m} / \mathrm{z}): 454.6972$ $\left([\mathrm{M}]^{+}\right), 423.6535\left([\mathrm{M}-\mathrm{OMe}]^{+}\right)$.

4OMe,b: Yield: $53 \%(\mathrm{Rf}=0.4) . \delta: 0.36(\mathrm{~s}, 6 \mathrm{H}, \mathrm{Si}-$ $\left.\mathrm{CH}_{3}\right), 0.72\left(\mathrm{t}, J=8.5 \mathrm{~Hz}, 4 \mathrm{H}, \mathrm{SiCH}_{2}\right), 1.58-1.75(\mathrm{~m}$, $\left.12 \mathrm{H}, \mathrm{SiCH}_{2}\left(\mathrm{CH}_{2}\right)_{3}\right),[2.39(\mathrm{q}, J=2.6 \mathrm{~Hz}), 2.67(\mathrm{q}$, $J=3.7 \mathrm{~Hz}), 4 \mathrm{H}, \mathrm{CH}_{2}$ in epoxy ring], $3.20(\mathrm{~m}, 2 \mathrm{H}$, $\mathrm{CH}$ in epoxy ring), $3.58\left(\mathrm{~s}, 6 \mathrm{H}, \mathrm{SiOCH}_{3}\right), 7.43$ (s, $4 \mathrm{H}, \mathrm{C}_{6} \mathrm{H}_{4}$ ).

4OMe,c: Yield: $39 \%(\mathrm{Rf}=0.4) . \delta: 0.37(\mathrm{~s}, 6 \mathrm{H}, \mathrm{Si}-$ $\left.\mathrm{CH}_{3}\right), 0.77$, (t, $\left.J=8.1 \mathrm{~Hz}, 4 \mathrm{H}, \mathrm{SiCH}_{2}\right), 1.08-2.16(\mathrm{~m}$, $18 \mathrm{H}, \mathrm{SiCH}_{2} \mathrm{CH}_{2}$ and $\mathrm{CH}_{2} \mathrm{CH}$ in cyclohexane ring), $3.10\left(\mathrm{~m}, 4 \mathrm{H}, \mathrm{CH}\right.$ in epoxy ring), $3.53\left(\mathrm{~s}, 6 \mathrm{H}, \mathrm{SiOCH}_{3}\right)$, $7.46\left(\mathrm{~s}, 4 \mathrm{H}, \mathrm{C}_{6} \mathrm{H}_{4}\right)$.

(Epoxy-functionalized alkyl)methyldimethoxysilanes $(5, a-c)$

A solution of $\mathrm{Pt}(0)(\mathrm{DVTMDS})_{2}$ in xylene $(0.01 \mathrm{~g}$, $36.0 \mu \mathrm{mol})$ was added to a mixture of dimethoxy-
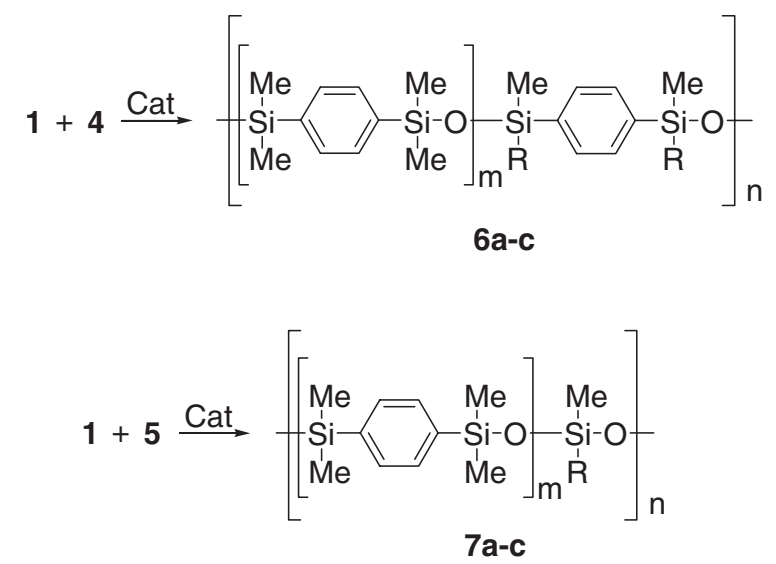

Scheme 3.

methylsilane $(3.82 \mathrm{~g}, 36.0 \mathrm{mmol})$ and AGE or $\mathrm{EH}$ or VCHE $(54.0 \mathrm{mmol})$ in THF. After stirring at $60^{\circ} \mathrm{C}$, the reaction mixture was filtered by a short florisil column. The products were purified by column chromatography.

5a: Yield: 53\% $(\mathrm{Rf}=0.5) . \delta: 0.07\left(\mathrm{~s}, 3 \mathrm{H}, \mathrm{SiCH}_{3}\right)$, 0.95 (t, $\left.J=7.0 \mathrm{~Hz}, 2 \mathrm{H}, \mathrm{SiCH}_{2}\right), 1.60\left(\mathrm{~m}, 2 \mathrm{H}, \mathrm{SiCH}_{2}-\right.$ $\left.\mathrm{CH}_{2}\right),[2.58(\mathrm{q}, J=2.7 \mathrm{~Hz}), 2.77(\mathrm{q}, J=4.6 \mathrm{~Hz}), 2 \mathrm{H}$, $\mathrm{CH}_{2}$ in epoxy ring], $3.11(\mathrm{~m}, 1 \mathrm{H}, \mathrm{CH}$ in epoxy ring), [3.36 (q, $J=3.1 \mathrm{~Hz}), 3.68(\mathrm{q}, J=3.2 \mathrm{~Hz}), 2 \mathrm{H}, \mathrm{Si}-$ $\left.\left(\mathrm{CH}_{2}\right)_{3} \mathrm{OCH}_{2}\right], 3.43\left(\mathrm{~m}, 2 \mathrm{H}, \mathrm{Si}\left(\mathrm{CH}_{2}\right)_{2} \mathrm{CH}_{2} \mathrm{O}\right), 3.57$ (s, $6 \mathrm{H}, \mathrm{SiOCH}_{3}$ ).

5,b: Yield: $44 \%(\mathrm{Rf}=0.7) . \delta: 0.08\left(\mathrm{~s}, 3 \mathrm{H}, \mathrm{SiCH}_{3}\right)$, $0.63\left(\mathrm{t}, J=7.7 \mathrm{~Hz}, 2 \mathrm{H}, \mathrm{SiCH}_{2}\right), 1.63-1.81(\mathrm{~m}, 6 \mathrm{H}$, $\left.\mathrm{SiCH}_{2}\left(\mathrm{CH}_{2}\right)_{3}\right),[2.44(\mathrm{q}, J=2.7 \mathrm{~Hz}), 2.67(\mathrm{q}, J=3.4$ $\mathrm{Hz}), 2 \mathrm{H}, \mathrm{CH}_{2}$ in epoxy ring], $3.28(\mathrm{~m}, 1 \mathrm{H}, \mathrm{CH}$ in epoxy ring), $3.57\left(\mathrm{~s}, 6 \mathrm{H}, \mathrm{SiOCH}_{3}\right)$.

5,c: Yield: 58\% $(\mathrm{Rf}=0.6) . \delta: 0.07\left(\mathrm{~s}, 3 \mathrm{H}, \mathrm{SiCH}_{3}\right)$, $0.69,\left(\mathrm{t}, J=7.3 \mathrm{~Hz}, 2 \mathrm{H}, \mathrm{SiCH}_{2}\right), 1.04-2.08(\mathrm{~m}, 9 \mathrm{H}$, $\mathrm{SiCH}_{2} \mathrm{CH}_{2}$ and $\mathrm{CH}_{2} \mathrm{CH}$ in cyclohexane ring), 3.11 $\left(\mathrm{m}, 2 \mathrm{H}, \mathrm{CH}\right.$ in epoxy ring), $3.48\left(\mathrm{~d}, 6 \mathrm{H}, \mathrm{SiOCH}_{3}\right)$.

\section{Polymerization}

Hetero-condensation routes with the combination of different functional groups with different catalyst were carried out as shown in Scheme 3.

Tin catalyst was selected as a classical catalyst, and tris(pentafluorophenyl)borane was used as a newly developed active catalyst. Typical examples were given.

\section{By Tris(pentafuluorophenyl)borane $e^{20}$}

To the mixture of $10 \mathrm{H}(0.42 \mathrm{~g}, 0.30 \mathrm{mmol})$ or $10 \mathrm{Me}$ $(0.44 \mathrm{~g}, 0.30 \mathrm{mmol})$ and $\mathbf{4 H}(0.30 \mathrm{mmol})$ in dry toluene $(5 \mathrm{~mL}), \mathrm{B}\left(\mathrm{C}_{6} \mathrm{~F}_{5}\right)_{3}(0.002 \mathrm{~g}, 3.00 \mu \mathrm{mol})$ was added as the catalyst. After reacting at room temperature for $5 \mathrm{~h}$, the polymer was precipitated into methanol.

For the case of prepolymer $1 \mathrm{H}(0.41 \mathrm{~g}, 0.30 \mathrm{mmol})$, monomer $40 \mathrm{OH}(0.30 \mathrm{mmol})$ and $40 \mathrm{Me}(0.30 \mathrm{mmol})$ were used. 
6a: Yield: $75 \% . \delta$ : $\left[0.32\left(\mathrm{~s}, \mathrm{Si}\left(\mathrm{CH}_{3}\right)_{2}\right), 0.38\right.$ (s, Si$\left.\mathrm{CH}_{3}\right)$, integral area ratio, 26/1], $0.84(\mathrm{t}, J=8.1 \mathrm{~Hz}$, $\left.\mathrm{SiCH}_{2}\right), 1.66\left(\mathrm{~m}, \mathrm{SiCH}_{2} \mathrm{CH}_{2}\right)$, [2.64 (q, $\left.J=2.7 \mathrm{~Hz}\right)$, $2.84(\mathrm{q}, J=4.6 \mathrm{~Hz}), \mathrm{CH}_{2}$ in epoxy ring], $3.14(\mathrm{~m}$, $\mathrm{CH}$ in epoxy ring), $3.34(\mathrm{q}, J=5.9 \mathrm{~Hz}), 3.68(\mathrm{q}, J=$ $\left.3.1 \mathrm{~Hz}), \mathrm{Si}\left(\mathrm{CH}_{2}\right)_{3} \mathrm{OCH}_{2}\right], 3.47\left(\mathrm{~m}, \mathrm{Si}\left(\mathrm{CH}_{2}\right)_{2} \mathrm{CH}_{2} \mathrm{O}\right)$, [7.53 (s, $\mathrm{C}_{6} \mathrm{H}_{4}$ in TMPS unit), 7.55 ( $\mathrm{s}, \mathrm{C}_{6} \mathrm{H}_{4}$ in functionalized unit), integral area ratio, 13/1]. $\mathrm{E}_{\mathrm{LEM}}$. $\mathrm{A}_{\mathrm{NAL}}$. Calcd for $\left(\left(\mathrm{C}_{10} \mathrm{H}_{16} \mathrm{Si}_{2} \mathrm{O}\right)_{13.0} \mathrm{C}_{20} \mathrm{H}_{32} \mathrm{Si}_{2} \mathrm{O}_{5}\right): \mathrm{C}$, $57.78 \%$, H, 7.76\%. Found: C, 57.57\%, H, 7.68\% (from 10H and 4H); C, 58.01, H, 7.84 (from 1H and 40H); C, 58.16, H, 7.81 (from 1H and 40Me).

6b: Yield: $80 \%$. $\delta$ : $0.52\left(\mathrm{t}, J=8.2 \mathrm{~Hz}, \mathrm{SiCH}_{2}\right), 1.61-$ $1.86\left(\mathrm{~m}, \mathrm{SiCH}_{2}\left(\mathrm{CH}_{2}\right)_{3}\right),[2.40(\mathrm{q}, J=2.6 \mathrm{~Hz}), 2.67$ (q, $J_{2}=3.6 \mathrm{~Hz}$ ), $\mathrm{CH}_{2}$ in epoxy ring], 3.20 (m, $\mathrm{CH}$ in epoxy ring). Other signals appeared at correspondent reasonable positions.

6c: Yield: $69 \%$. $\delta$ : 0.73, (t, $\left.J=8.5 \mathrm{~Hz}, \mathrm{SiCH}_{2}\right), 1.08-$ $2.23\left(\mathrm{~m}, \mathrm{SiCH}_{2} \mathrm{CH}_{2}\right.$ and in cyclohexane ring), 3.12 ( $\mathrm{m}, \mathrm{CH}$ in epoxy ring). Other signals appeared at correspondent positions.

In the synthesis of polymer 7, since the monomers 5 with $-\mathrm{H}$ and $-\mathrm{OH}$ functional groups were difficult to get, we used hetero-polycondesation polymerization of $1 \mathbf{H}$ with 5 having -OMe terminal group in the presence of tin catalyst.

7a: Yield: 85\%. $\delta$ : $\left[0.07\left(\mathrm{~s}, \mathrm{SiCH}_{3}\right), 0.32\right.$ (s, Si$\left.\left(\mathrm{CH}_{3}\right)_{2}\right)$, integral area ratio, $\left.1 / 26\right], 0.88(\mathrm{t}, J=7.1 \mathrm{~Hz}$, $\mathrm{SiCH}_{2}$ ), $1.64\left(\mathrm{~m}, \mathrm{SiCH}_{2} \mathrm{CH}_{2}\right)$, [2.61 (q, $\left.J=2.7 \mathrm{~Hz}\right)$, $2.79(\mathrm{q}, J=4.6 \mathrm{~Hz}), \mathrm{CH}_{2}$ in epoxy ring], $3.15(\mathrm{~m}$, $\mathrm{CH}$ in epoxy ring), [3.30 (q, $J=3.0 \mathrm{~Hz}), 3.75(\mathrm{q}, J=$ $\left.3.1 \mathrm{~Hz}), \mathrm{Si}\left(\mathrm{CH}_{2}\right)_{3} \mathrm{OCH}_{2}\right], 3.44\left(\mathrm{~m}, \mathrm{Si}\left(\mathrm{CH}_{2}\right)_{2} \mathrm{CH}_{2} \mathrm{O}\right)$, $7.53\left(\mathrm{~s}, \mathrm{C}_{6} \mathrm{H}_{4}\right)$. $\mathrm{E}_{\mathrm{LEM}}$. $\mathrm{A}_{\mathrm{NAL}}$. Calcd for $\left(\mathrm{C}_{10} \mathrm{H}_{16^{-}}\right.$ $\left.\mathrm{Si}_{2} \mathrm{O}\right)_{13.0} \mathrm{C}_{7} \mathrm{H}_{14} \mathrm{SiO}_{3}$ ): C, 57.07\%, H, 7.76\%. Found: C, $57.35 \%, \mathrm{H}, 7.80 \%$.

7b: Yield: $89 \%$. $\delta$ : $0.52\left(\mathrm{t}, J=7.2 \mathrm{~Hz}, \mathrm{SiCH}_{2}\right), 1.47-$ $1.74\left(\mathrm{~m}, \mathrm{SiCH}_{2}\left(\mathrm{CH}_{2}\right)_{3}\right), 2.33(\mathrm{q}, J=2.6 \mathrm{~Hz}), 2.56$ (q, $J=3.4 \mathrm{~Hz}$ ), $\mathrm{CH}_{2}$ in epoxy ring], 3.05 (m, $\mathrm{CH}$ in epoxy ring). Other signals appeared at correspondent reasonable positions.

7c: Yield: $64 \%$. $\delta$ : 0.74, (t, $\left.J=7.4 \mathrm{~Hz}, \mathrm{SiCH}_{2}\right), 1.09-$ 2.22 (m, $\mathrm{SiCH}_{2} \mathrm{CH}_{2}$ and $\mathrm{CH}_{2} \mathrm{CH}$ in cyclohexane ring), 3.18 ( $\mathrm{m}, \mathrm{CH}$ in epoxy ring). Other signals appeared at correspondent positions.

\section{By Di-n-butyltin Dilaurate}

Di- $n$-butyltin dilaurate $(0.002 \mathrm{~g}, 3.00 \mu \mathrm{mol})$ was added to a mixture of prepolymer $\mathbf{1 0 H}(0.42 \mathrm{~g}, 0.30$ mmol) or 10Me $(0.44 \mathrm{~g}, 0.30 \mathrm{mmol})$ and monomer 40Me or $40 \mathrm{OH}(0.30 \mathrm{mmol})$ in $\mathrm{THF}$. After stirring at $40^{\circ} \mathrm{C}$, the reaction mixture was filtered by a short Florisil column to separate the catalysts. Removal of the solvent gave the products. The products were purified by reprecipitation from toluene into methanol. The yield of polymers were 34-60\%.

\section{Formation of Film and Cross-Linking}

Polymer 6a $\left(M_{\mathrm{n}}=6,400,0.50 \mathrm{~g}, 0.32 \mathrm{mmol}\right.$ for epoxy group) obtained by tris(pentafluorophenyl)borane-catalyzed polymerization and 1,4-diaminobenzene $(0.017 \mathrm{~g}, 0.16 \mathrm{mmol}-0.0086 \mathrm{~g}, 0.080 \mathrm{mmol})$ were dissolved in toluene $(5 \mathrm{~mL})$, and cast to obtain a film. When it was difficult to obtain a film directly, the solution was heated at $80^{\circ} \mathrm{C}$ for $5 \mathrm{~h}$, then cast, this is the general case. The film was heat-treated further at $80^{\circ} \mathrm{C}$ for $24 \mathrm{~h}$ under nitrogen.

FT-IR (neat, $\mathrm{cm}^{-1}$ ): 669, 697, 736, 796, 1080, 1138, 1199, 1260, 1357, 1380, 2956, 3629, 3723.

\section{RESULTS AND DISCUSSION}

\section{Synthesis and Characterization}

\section{Prepolymers}

Poly(silphenylenesiloxane)s have been generally synthesized by homo-condensation reaction of phenylenedisilanol, or hetero-condensation of disilanol with suitable difunctional compopund, having chloro-, acetoxy-, amino-, ureido-, ${ }^{21,22}$ or alkoxy- groups. When two different silanols are used, obstacle is often encountered by the contamination of homo-condensed product in the hetero-condensed product, which significantly damages the control of the regular structure of the formed polymers.

To improve the selectivity of hetero-condensation by averting side reactions, PTMPS prepolymers with three different terminals, hydroxy, methoxy and hydro groups, were prepared starting from BSB for hetero-polycondensation reaction. By using slightly excess amount of water in the palladium-catalyzed dehydrocoupling polymerization of $\mathrm{BSB},{ }^{21,23,24}$ followed by careful fractional precipitation, prepolymer $\mathbf{1 0 H}$ with narrow polydispersity was obtained. In the case of prepolymers $\mathbf{1 H}$, it was difficult to obtain a prepolymer with controlled high molecular weight from BSB directly (see Table I), and the prepolymer with hydro terminal was obtained by LAH reduction. Prepolymer 10Me was obtained by Pd-catalyzed methanolysis of $\mathbf{1 H}$. Molecular characteristics of prepolymers are summarized in Table I.

All the prepolymers gave reasonable elemental analysis data, when the low molecular weight of the prepolymer is considered. Number-average molecular weight was determined by ${ }^{1} \mathrm{H}$ NMR for $\mathbf{1 H}$. The values by SEC and ${ }^{1} \mathrm{H}$ NMR are quite close. Both prepolymers, 1H and 10Me had almost the same degree of polymerization and narrow polydispersities with the starting $\mathbf{1 0 H}$, determined by SEC, which indicated almost no degradation reaction had occurred during the functional transformation from $\mathrm{SiOH}$ to $\mathrm{Si}$ methoxy group through $\mathrm{SiH}$. 
Table I. Molecular characteristics of prepolymers and polymers with epoxy side groups

\begin{tabular}{|c|c|c|c|c|}
\hline \multirow{2}{*}{$\begin{array}{l}\text { Prepolymers and } \\
\text { polymers }\end{array}$} & \multicolumn{2}{|c|}{ Molecular weight ${ }^{\mathrm{a}}$} & \multirow{2}{*}{ PDI } & \multirow{2}{*}{$\mathrm{m} / \mathrm{n}$} \\
\hline & $M_{\mathrm{w}} \times 10^{3}$ & $M_{\mathrm{n}} \times 10^{3}$ & & \\
\hline 1H & 2.8 & $2.7(2.72)^{\mathrm{c}}$ & 1.04 & $\begin{array}{c}13 /- \\
(13.0 /-)^{c}\end{array}$ \\
\hline $1 \mathbf{H}^{* \mathrm{~b}}$ & 1.6 & 1.6 & 1.01 & $8 /-$ \\
\hline $10 \mathrm{H}$ & 2.9 & 2.8 & 1.04 & $13 /-$ \\
\hline 10Me & 3.0 & 2.9 & 1.03 & $13 /-$ \\
\hline $6 \mathbf{a}$ & 11.6 & 6.4 & 1.81 & $13 / 2$ \\
\hline $\mathbf{6 b}$ & 17.8 & 9.8 & 1.80 & $13 / 3$ \\
\hline $\mathbf{6 b}^{* d}$ & 9.9 & 6.2 & 1.59 & $8 / 3$ \\
\hline $6 c$ & 12.5 & 6.8 & 1.83 & $13 / 2$ \\
\hline $7 \mathbf{a}$ & 18.6 & 13.2 & 1.40 & $13 / 5$ \\
\hline $7 b$ & 19.8 & 17.8 & 1.11 & $13 / 6$ \\
\hline $7 c$ & 14.1 & 9.9 & 1.41 & $13 / 3$ \\
\hline PTMPS & 15.3 & 12.4 & 1.23 & - \\
\hline
\end{tabular}

${ }^{a}$ Determined by SEC in relation with polystyrene standard. ${ }^{b}$ Low molecular weight oligomer was prepared by changing the ratio of $\mathrm{SiH}$ and water. ${ }^{\mathrm{c} C a l c u l a t e d}$ by ${ }^{1} \mathrm{H}$ NMR spectrum.

${ }^{\mathrm{d}}$ Starting from low molecular weight oligomer.

\section{Monomers}

Mmonomers containing hydro, hydroxy, and methoxy groups were synthesized starting from 1,4-dibromobenzene. Since only one $\mathrm{Si}-\mathrm{Cl}$ group of the starting dichlorosilanes is required to be consumed in the Grignard reaction, excess dichlorosilanes were used in the synthesis of $\mathbf{2}$. The reaction was controlled to proceed at room temperature within a relatively short reaction time in order to minimize the unexpected disproportionation reaction of the silane derivatives, which is often observed in such reactions involving silanes containing at least one hydro- substituent. $^{23-25}$

Compound 3 with silane terminal was synthesized by the reduction with LAH. This compound was stable enough in air, and can be used to introduce various functional groups.

Compounds $\mathbf{4}$ and $\mathbf{5}$ were prepared by hydrosilylation reaction. Compounds 4's with hydroxy and methoxy functional groups were obtained by hydrolysis and methanolysis reaction, respectively. EI-MS analysis of the compounds $\mathbf{4 a}$ indicated the complete conversion of silane group to epoxy-containing side groups.

\section{Polymerization}

Hetero-polycondensation reactions were carried out in the presence of tin and borane catalysts. Tin catalyst gave lower yield of the products (39-58\%). The process to remove catalyst might be one reason to reduce the yield. ${ }^{1} \mathrm{H}$ NMR spectra of the polymers prepared under different conditions are compared in Table II and Figure 1.
Table II. Influence of catalyst in the synthesis of poly(silphenylenesiloxane) $\mathbf{6 a}$

\begin{tabular}{|c|c|c|c|c|}
\hline No. & Catalysts & $\begin{array}{l}\text { Reaction } \\
\text { temperature } \\
\left({ }^{\circ} \mathrm{C}\right)\end{array}$ & $\begin{array}{l}\text { Time } \\
\text { (h) }\end{array}$ & $\begin{array}{l}\text { Yield } \\
(\%)\end{array}$ \\
\hline 1) & $\mathrm{B}\left(\mathrm{C}_{6} \mathrm{~F}_{5}\right)_{3}$ & r.t. & 5 & 87 \\
\hline 2) & $\begin{array}{l}\text { di- } n \text {-butyltin } \\
\text { dilaurate }\end{array}$ & 40 & 8 & 58 \\
\hline 3) & $\begin{array}{l}\text { di-n-butyltin } \\
\text { dilaurate }\end{array}$ & 40 & 8 & 45 \\
\hline 4) & $\mathrm{B}\left(\mathrm{C}_{6} \mathrm{~F}_{5}\right)_{3}$ & r.t. & 5 & 89 \\
\hline 5) & $\mathrm{B}\left(\mathrm{C}_{6} \mathrm{~F}_{5}\right)_{3}$ & r.t. & 5 & 73 \\
\hline 6) & $\mathrm{B}\left(\mathrm{C}_{6} \mathrm{~F}_{5}\right)_{3}$ & r.t. & 5 & 61 \\
\hline 7) & $\begin{array}{c}\text { di- } n \text {-butyltin } \\
\text { dilaurate }\end{array}$ & 40 & 8 & 39 \\
\hline
\end{tabular}

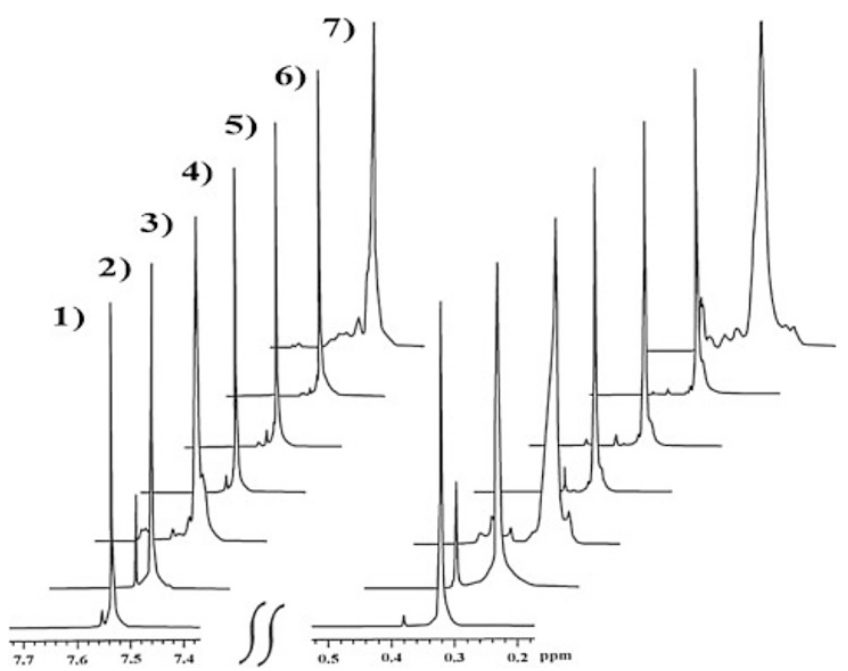

Figure 1. ${ }^{1} \mathrm{H}$ NMR spectra of polymer 6a in the methyl and phenyl regions obtained in the hetero-condensation polymerization systems by the combination of:

1) $10 \mathrm{H}, 4 \mathrm{H}, \mathrm{a}$; 2) $1 \mathrm{OH}, 40 \mathrm{H}, \mathrm{a}$; 3) $10 \mathrm{H}, 40 \mathrm{Me}, \mathrm{a}$; 4) $1 \mathrm{H}, 4 \mathrm{OH}$, a; 5) $1 \mathrm{H}, 40 \mathrm{Me}, \mathrm{a}$; 6 ) 10Me, 4H, a; 7) 10Me, 40H, a.

Combination of functional groups of hydroxy and methoxy in either monomer or prepolymer, catalyzed by tin gave polymers with broad methyl and phenyl signals at 0.33 and $7.55 \mathrm{ppm} \mathrm{[3)} \mathrm{and} \mathrm{7)} \mathrm{in} \mathrm{Figure} \mathrm{1]}$ with extra peaks. Especially in 7), strong peak was seen at 0.42 and $7.57 \mathrm{ppm}$ assignable to $40 \mathrm{OH}$ unit, which was considerably stronger than the calculated value as an ideal copolymer. Homo-condensation of functionalized monomer 4 seemed to have been caused by tin catalyst, resulting in various non-controlled chemical structure of the formed polymer. Combination of silanol and silanol catalyzed by tin [2) in Figure 1] gave sharper methyl signal, but considerably strong signal was again seen at $0.42 \mathrm{ppm}$, indicating the occurrence of homo-condensation of 4 .

When silane functional group was used in either monomer or prepolymer, a clean and sharp methyl 


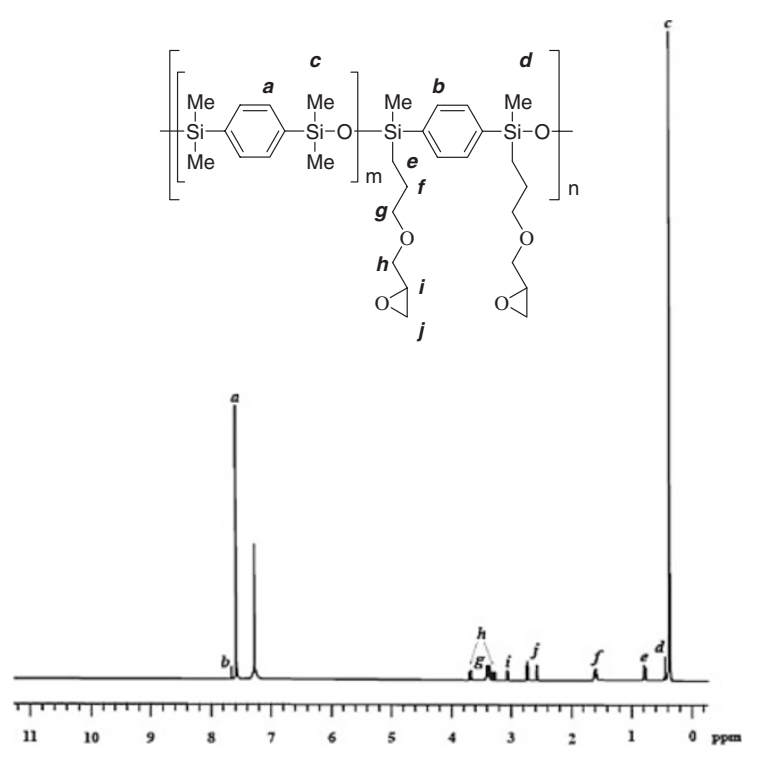

Figure 2. ${ }^{1} \mathrm{H}$ NMR spectrum of $\mathbf{6 a}$.

and phenyl signals with ideal area ratio was obtained in higher yield even at room temperature (No. 1: 87; No. 4: $89 \%$ in Table II) by the borane catalyst. The integral ratio of peaks at 0.42 and $7.57 \mathrm{ppm}$ to those at 0.33 and $7.55 \mathrm{ppm}$ is consistent with the feed ratio of the prepolymer and monomer by considering the degree of polymerization of the prepolymer. No homo-condensation of $\mathbf{1 0 H}$ was observed (No. 1). Elemental analysis of the polymer reasonably well coincided with the calculated value within experimental error. Elemental analysis of the polymer from $\mathbf{1 H}$ and $\mathbf{4 O H}, \mathbf{a}$ deviated a little more from the calculated value. Homo-condensation of $\mathbf{4 0 H}, \mathbf{a}$ might have occurred to a small extent (No. 4). Homocondensation of $\mathbf{4 , a}$ were completely suppressed by using 40Me, a (No. 5), or $\mathbf{4 H}$, a (No. 6). However, the yield was rather lower in these combinations compared with No. 1 and No. 4, and the deviation of the elemental analysis was a little bigger. The ${ }^{1} \mathrm{H}$ NMR signal contained small impurity peaks. Some unknown side reaction might have occurred. So, we chose tris(pentafluorophenyl)borane as an excellent catalyst to synthesize polymers with regulated sequence using the combination of $\mathbf{1 0 H}$ and $\mathbf{4 H}$.

The hetero-condensation polymerization of prepolymer $\mathbf{1}$ with compounds $\mathbf{4}$ and $\mathbf{5}$ gave smoothly polymer $\mathbf{6}$ and $\mathbf{7}$. The yield was rather poor for cyclohexene oxide substituted $\mathbf{4 c}$ or $\mathbf{5 c}$. Some steric hindrance might be operating. Typical spectra were given for $6 \mathbf{a}$ and 7a (Figures 2 and 3.).

Distinct difference in ${ }^{1} \mathrm{H}$ NMR between 6a and 7a can be observed at methyl signals as shown in Figure 3. Methyl protons in functionalized unit of polymer $\mathbf{6 a}$ appeared at $0.41 \mathrm{ppm}$, and that of polymer 7a appeared at $0.07 \mathrm{ppm}$. This fact also supports the

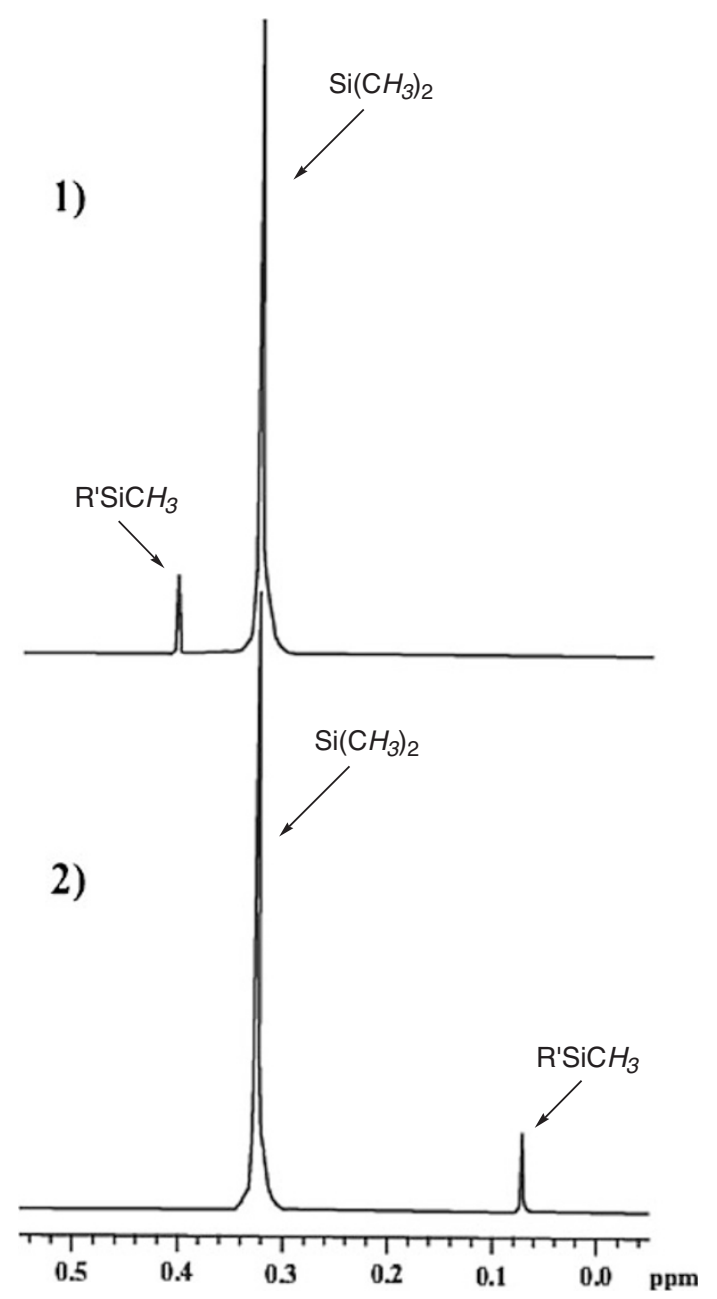

Figure 3. ${ }^{1} \mathrm{H}$ NMR spectra of the methyl region of 1) (polymer 6a) and 2) (polymer 7a).

complete alternative structure of the polymers 6a and 7a. Thus, NMR confirmed the exactly alternating structure of polymer $\mathbf{6 a}$ and $7 \mathbf{a}$. The functional units are very well dispersed in the polymer chain.

Polymers with epoxy groups, $\mathbf{6}$ and $\mathbf{7}$, are white powder after precipitation in methanol at room temperature. Characteristic data of the polymers are also summarized in Table I.

It was established that the tris(pentafluorophenyl)borane was an excellent catalyst to synthesize PTMPS with controlled amounts of epoxy groups in regular structure.

\section{Cross-Linking}

The epoxy functional groups can be used to form cross-linked structures, which can be coat on the surface of the materials to make the films. Cross-linked poly(silphenylenesiloxane) was obtained from $\mathbf{6 a}$ and 1,4-diaminobenzene as a cross-linker. The slightly cross-linked film was further cross-linked by heating for $18 \mathrm{~h}$ at $80^{\circ} \mathrm{C}$ to give film with improved mechanical strength (self standing stretchable). 
In 6a, cross-linked (1:1) system, obtained with the 1:1 molar ratio of monomer unit and 1,4-diaminobenzene ( $1: 1$ for epoxide and $p-\mathrm{NH} ; 1$ to 2 for epoxide and all $\mathrm{NH}$ ), the $\mathrm{NH}$ stretching of primary amino group in the initially mixed polymer system at 3420 and $3580 \mathrm{~cm}^{-1}$ became weaker by preheating, and became to a further weaker peak at $3345 \mathrm{~cm}^{-1}$ in polymer film, and new broad peak appeared at around $3550-3700 \mathrm{~cm}^{-1}$ assignable to $\mathrm{OH}$ stretching. This indicated the opening of epoxy ring, but the existence of remaining primary amino group, which was an evidence for the existence of free primary amino group on one terminal of 1,4-diaminobenzene, suggested incomplete cross-linking of the system.

In cross-linked 6a, cross-linked (2:1) obtained with 2:1 molar ratio for epoxy and amino functional groups (1:1 for epoxide and $\mathrm{NH})$, the $\mathrm{NH}$ stretching of primary amino group has almost completely disappeared. The cross-linking reaction seemed to have occurred, unfortunately, characteristic $\mathrm{CH}$ stretching vibration of epoxy ring to determine the extent of the reaction of epoxy group could not be clearly identified because of the overlapping with aromatic $\mathrm{CH}$ stretching.

\section{Melting and Thermal Degradation Behavior}

Heat of melting and thermal degradation behavior of prepolymers, polymers and cross-linked polymers are shown in Table III.

The prepolymers showed melting points at around $80-110^{\circ} \mathrm{C}$. The $\Delta H_{\text {melt }}$ was similar to that of PTMPS $\left(T_{\mathrm{g}}=-22^{\circ} \mathrm{C}, T_{\mathrm{m}}=110.8^{\circ} \mathrm{C}\right)$. They decomposed at around $330-340{ }^{\circ} \mathrm{C}$ in air and $370-395^{\circ} \mathrm{C}$ in nitrogen.

Introduction of epoxide groups through tetramethylene spacer $(1 / 14$ and $1 / 9$ per phenylenedisiloxane repeating unit in prepolymer $1 \mathbf{H}$ and $\mathbf{1 H}^{*}$ ) to the polymer structure ( $6 \mathbf{b}$ and $\left.6 \mathbf{b}^{*}\right)$ increased the melting temperature a little, but reduced the melting enthalpy to around $20 \mathrm{~J} / \mathrm{g}$ from $42.5 \mathrm{~J} / \mathrm{g}$ of non-modified polymer (PTMPS). The polymers did not show $T_{\mathrm{g}}$ at around $-40 \sim-20^{\circ} \mathrm{C}$. Introduction of epoxide through trimethyleneoxymethylene spacer, or cyclohexene oxide through ethylene spacer showed only small effects on the property of the polymers $\mathbf{6 a}$ and $\mathbf{6 c}$. Introduction of siloxane linkage to the polymer structure (7) slightly influenced the property of the polymer.

TGA curves of polymers under nitrogen atmosphere and in air at a heating rate of $10^{\circ} \mathrm{C} / \mathrm{min}$ are shown in Figure 4.

It can be seen that a smooth single-step degradation process occurred in nitrogen, but a little complex multi-step processes in air. The results were also summarized in Tables III.

Poly(silphenylenedsiloxane)s having epoxy side groups generally exhibited lower degradation onset
Table III. Heat of melting and thermal degradation behaviors of prepolymers, polymers and cross-linked polymers

\begin{tabular}{|c|c|c|c|c|c|c|}
\hline \multirow[t]{2}{*}{ Compounds } & \multirow[t]{2}{*}{$\begin{array}{l}T_{\mathrm{m}}{ }^{\mathrm{a}} \\
\left({ }^{\circ} \mathrm{C}\right)\end{array}$} & \multirow[t]{2}{*}{$\begin{array}{c}\Delta H_{\text {melt }} \\
(\mathrm{J} / \mathrm{g})\end{array}$} & \multicolumn{2}{|c|}{$\begin{array}{c}\text { Onset of } \\
\text { degradation } \\
T_{\text {d,onset }}\left({ }^{\circ} \mathrm{C}\right)^{\mathrm{b}}\end{array}$} & \multicolumn{2}{|c|}{$\begin{array}{c}\text { Residue at } \\
800^{\circ} \mathrm{C} \\
(\%)\end{array}$} \\
\hline & & & In air & In $\mathrm{N}_{2}$ & In air & In $\mathrm{N}_{2}$ \\
\hline $1 \mathrm{H}$ & 89.0 & 46.8 & 337 & 394 & 32 & 30 \\
\hline $1 \mathbf{H}^{* \mathrm{c}}$ & 78.4 & 47.3 & 330 & 370 & 34 & 25 \\
\hline $10 \mathrm{H}$ & 110.0 & 48.2 & 341 & 381 & 37 & 33 \\
\hline 10Me & 95.6 & 46.9 & 334 & 374 & 31 & 30 \\
\hline $6 a$ & 119.3 & 28.4 & 374 & 425 & 36 & 30 \\
\hline $\mathbf{6 b}$ & 118.1 & 21.2 & 375 & 452 & 43 & 37 \\
\hline $6 b^{* d}$ & 104.6 & 25.2 & 310 & 327 & 29 & 32 \\
\hline $6 c$ & 108.5 & 18.8 & 353 & 364 & 34 & 30 \\
\hline $7 \mathbf{a}$ & 115.8 & 35.4 & 359 & 310 & 36 & 30 \\
\hline $7 b$ & 122.6 & 35.0 & 364 & 350 & 44 & 35 \\
\hline $7 c$ & 110.6 & 27.3 & 319 & 300 & 31 & 34 \\
\hline $\begin{array}{c}\text { 6a,cross-limked } \\
(1: 1)\end{array}$ & - & - & 450 & 490 & 65 & 37 \\
\hline $\begin{array}{c}\text { 6a,cross-limked } \\
(2: 1)\end{array}$ & - & - & 421 & 470 & 60 & 40 \\
\hline PTMPS & 110.8 & 42.5 & 390 & 423 & 31 & 37 \\
\hline
\end{tabular}

${ }^{\mathrm{a}}$ Determined by DSC. ${ }^{\mathrm{b}}$ The temperature at $5 \%$ weight loss. ${ }^{\mathrm{c}, \mathrm{d}}$ See comments b, c in Table I.

temperature than PTMPS, probably by the enhanced degradation initiated by the ring-opening of epoxy functional groups. Glycidyloxylpropyl and 3-(2,3epoxycyclohexyl-1-yl) groups seemed to be the most easily degraded among the epoxy groups studied. Nevertheless, it is noticeable that the polymer $\mathbf{6 b}$ with tetramethylene spacer groups showed the highest, or close to the highest onset temperature of degradation and residual yield both in air $\left(375^{\circ} \mathrm{C}, 43 \%\right)$ and nitrogen $\left(452^{\circ} \mathrm{C}, 37 \%\right)$.

The polymers seemed to have completely degraded during the heating process in both air and nitrogen and showed similar residual yield with PTMPS except for 6b and $\mathbf{7 b}$ in air. After undergoing thermal degradation process in air, the residue of every polymer is white hard solid, which looks quite different from the black residue under nitrogen. ${ }^{9}$ The white residue is considered to be silica formed from the thermooxidative degradation in air. Polymer $\mathbf{6} \mathbf{b}^{*}$ with higher concentration of epoxy group showed the lower onset temperature. Certain length of silphenylene siloxane units without perturbation of the structure seems to be necessary.

The cross-linked film 6a,cross-linked (1:1) showed the onset temperature of degradation at $450{ }^{\circ} \mathrm{C}$ in air and $490{ }^{\circ} \mathrm{C}$ in nitrogen, which were much higher than those of 6a. The residual yield in air $(65 \%)$ became considerably higher than that of non-cross-linked polymer. Thermal properties of $\mathbf{6 a}$, cross-linked (1:1) and 6a,cross-linked (2:1) were similar. 


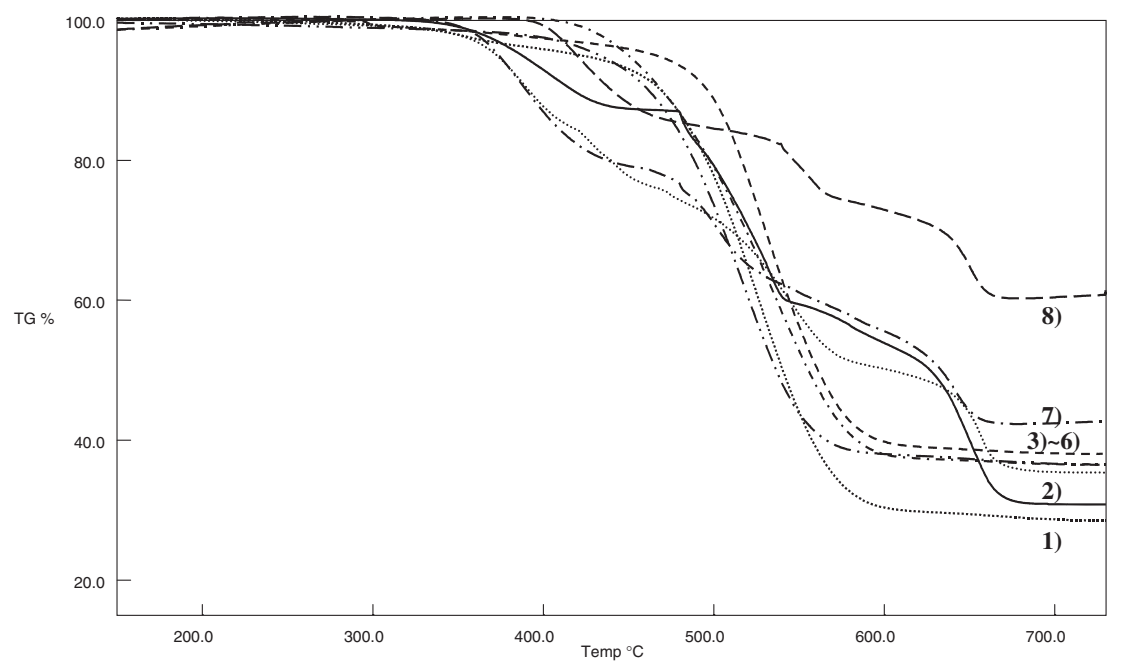

Figure 4. TGA* curves of poly(silphenylenesiloxane)s with epoxy side groups in air and in nitrogen. ${ }^{*}$ Measurements were carried out with a heating rate of $10^{\circ} \mathrm{C} / \mathrm{min}$ on about $5 \mathrm{mg}$ sample.

1) - - 6a in nitrogen;

2) - PTMPS in air;

3) … 6a in air;

4) -.. PTMPS in nitrogen;

5).$--6 b$ in nitrogen;

6) --6 a,cross-linked $(2: 1)$ in nitrogen;

7) -. $6 \mathrm{~b}$ in air;

8) -6 a,cross-linked $(2: 1)$ in air.

\section{CONCLUSIONS}

Poly(silphenylenesiloxane)s containing epoxy functional side groups were successfully synthesized by polycondensation of silane, silanol, and methoxysilane functional groups of silphenylenesiloxane prepolymer and monomers with epoxy functional groups. Tris(pentafluorophenyl)borane was the most suitable catalyst in the hetero polycondensation reactions when silane functional group was used in monomer together with prepolymer having silanol terminal groups. Cross-linking of the film gave self-supporting film with higher thermal stability.

Acknowledgment. This work was partly supported by a Grant-in-Aid for Scientific Research (16205016) from Japan Society for the Promotion of Science. This work was also partly supported by a Grant-in-Aid for Scientific Research on Priority Area (18039012) and City Area Program from the Ministry of Education, Science, Sports, Culture and Technology, Japan.

\section{REFERENCES}

1. M. Lewin, S. M. Atlas, and E. M. Pearce, "Flame Retardant Polymeric Materials," Plenum Press, New York, 1975.

2. P. R. Dvornic and R. W. Lenz, "High Temperature Siloxane
Elastomers," Hüthig \& Wepf, Basel, 1990.

3. "Silicon-Based Polymer Science: A Comprehensive Resource," J. M. Zeigler and G. F. W. Fearon, Ed., American Chemical Society, Washington, DC, 1990.

4. R. Corriu, D. Leclercq, P. H. Mutin, H. Samson, and A. Vioux, J. Polym. Sci., Part A: Polym. Chem., 32, 187 (1994).

5. L. W. Breed, R. L. Elliott, and M. E. Whitehead, J. Polym. Sci., Part A-1, 5, 2745 (1967).

6. R. E. Burks, E. R. Covington, M. V. Jackson, and J. E. Curry, J. Polym. Sci., Polym. Chem. Ed., 11, 319 (1973).

7. C. U. Pittman, W. J. Patterson, and S. P. Mcmanus, J. Polym. Sci., Polym. Chem. Ed., 14, 1715 (1976).

8. P. R. Dvornic and R. W. Lenz, J. Polym. Sci., Polym. Chem. $E d ., 20,593$ (1982).

9. P. R. Dvornic and R. W. Lenz, Polymer, 24, 763 (1983).

10. P. R. Dvornic, H. J. Perpall, P. C. Uden, and R. W. Lenz, J. Polym. Sci., Part A: Polym. Chem., 27, 3503 (1989).

11. Y. Liu, I. Imae, and Y. Kawakami, Polym. Int., 53, 1259 (2004).

12. J. S. Chen, C. K. Ober, M. D. Poliks, Y. Zhang, U. Wiesner, and C. Cohen, Polymer, 45, 1939 (2004).

13. T. M. Lee, C. C. M. Ma, C. W. Hsu, and H. L. Wu, Polymer, 46, 8286 (2005).

14. R. H. Baney, M. Itoh, A. Sakakibara, and T. Suzuki, Silsesquioxanes, Chem. Rev., 95, 1409 (1995).

15. A. Provatas and J. G. Matisons, TRIP, 5, 10 (1997).

16. J. D. Lichtenhan, Comments Inorg. Chem., 17, 115 (1995).

17. Y. Li and Y. Kawakami, Macromolecules, 32, 3540 (1999).

18. Y. Li and Y. Kawakami, Macromolecules, 32, 8768 (1999).

19. R. L. Merker and M. J. Scott, J. Polym. Sci., Part A: Polym. 
Chem., 2, 15 (1964).

20. D. Zhou and Y. Kawakami, Macromolecules, 38, 6902 (2005).

21. P. R. Dvornic and R. W. Lenz, J. Polym. Sci., Polym. Chem. Ed., 20, 951 (1982).

22. P. R. Dvornic and R. W. Lenz, Macromolecules, 25, 3769 (1992).
23. D. L. Bailey and N. Y. Buffalo, U.S. Patent 2723983 (1955); 2723984 (1955); 2745860 (1956).

24. K. Kabeta, S. Wakamatsu, and T. Imai, J. Polym. Sci., Part A: Polym. Chem., 34, 2991 (1996).

25. M. Itoh, K. Inoue, J. Ishikawa, and K. Iwata, J. Organomet. Chem., 629, 1 (2001). 